V. N. Karazin Kharkiv National

University

Shupyk National Medical Academy of

Postgraduate Education

Ukraine

\title{
EFFECTS OF IODINE SUPPLEMENTATION ON PHYSICAL AND PSYCHOMOTOR DEVELOPMENT IN YOUNG CHILDREN AND THEIR NEUROLOGICAL STATUS
}

\author{
Mamenko Marina \\ Professor, MD, PhD, Doctor of Medical Sciences \\ Dean of Pediatric Faculty \\ Shupyk National Medical Academy of Postgraduate Education \\ Shlieienkova Hanna \\ Assistant Professor, MD, PhD, \\ Department of Internal Medicine \\ V. N. Karazin Kharkiv National University
}

EAP-2017

Ljubljana, Slovenia 


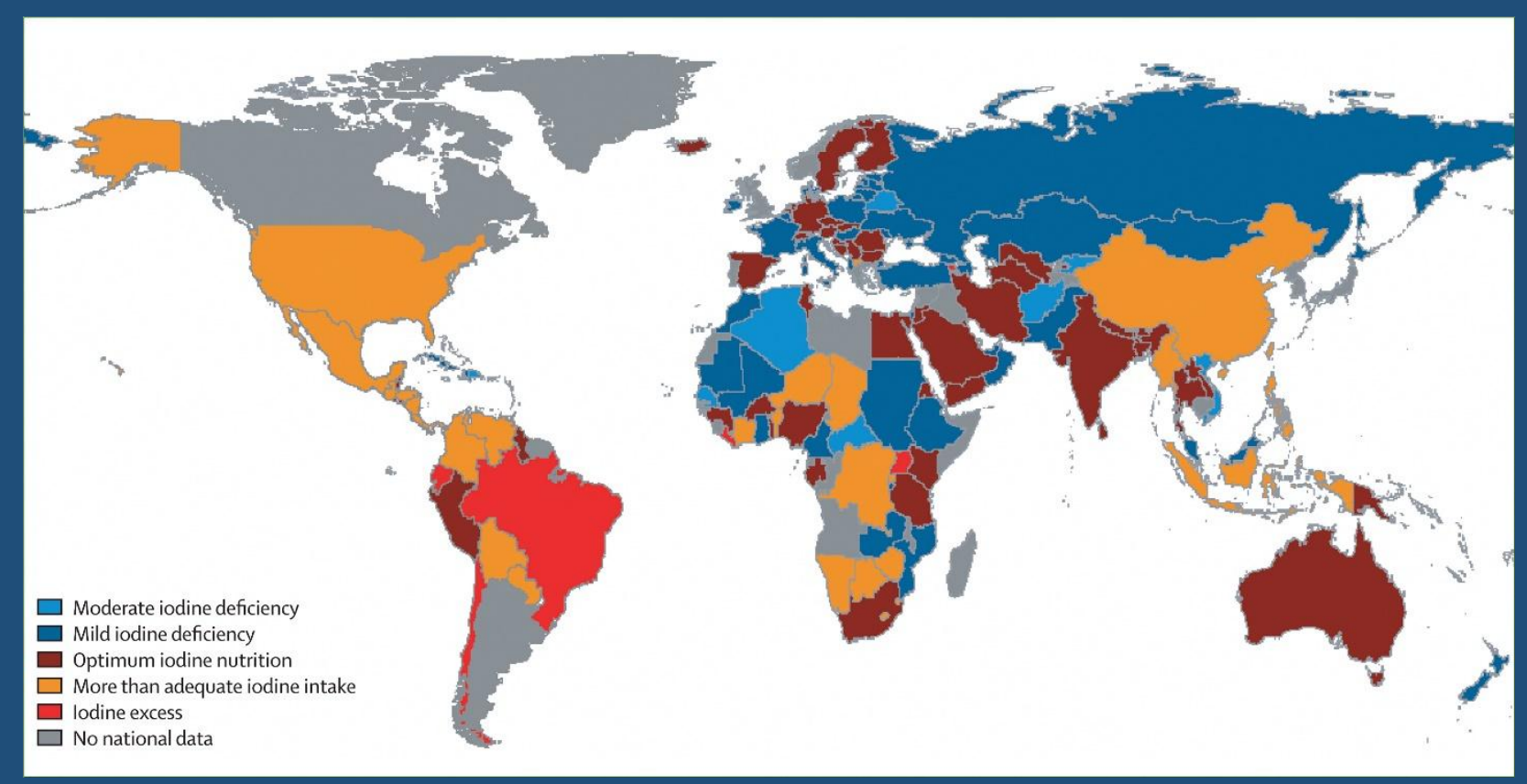

\section{Actuality}

- Iodine deficiency is the world's most prevalent, yet easily preventable, cause of brain damage.

- lodine deficiency disorders (IDD), which can start before birth, jeopardize children's mental health and often their very survival.

- lodine deficiency (ID) during pregnancy and infancy may impair growth and neurodevelopment, increase infant mortality. ID during childhood reduces somatic growth and cognitive and motor function.

- Greater significance is IDD's less visible, yet pervasive, mental impairment that reduces intellectual capacity at home, in school and at work.

\section{Aim}

To evaluate effects of iodine supplementation on physical and psychomotor development in young children and their neurological status

\section{Methods}

Target group: 118 children 0-3 years old from orphanage Methods:

- Examination physical and neuropsychological development

- Dietary iodine intake evaluation by urinary iodine concentration (Sandell-Kolthoff reaction)

- Thyroid status by TSH, T4, T3 serum-based measuring (IMA)

- lodine intake: infant formula (iodine concentration $100 \mu \mathrm{g} / \mathrm{l}$ ) -61 children (KI-) and additional iodine supplementation in 57 children $(\mathrm{KI}+)$ by drugs (50 $\mu \mathrm{g}$ per day for 6 months) 


\section{Results}

Impact of iodine supplementation on thyroid status in young children

\begin{tabular}{|c|c|c|c|c|c|c|c|c|c|}
\hline \multirow{2}{*}{\multicolumn{2}{|c|}{ Labs }} & \multicolumn{2}{|c|}{3 Months } & \multicolumn{2}{|c|}{6 Months } & \multicolumn{2}{|c|}{9 Months } & \multicolumn{2}{|c|}{12 Months } \\
\hline & & $\begin{array}{l}\mathrm{Kl}+ \\
(\mathrm{n}=57)\end{array}$ & $\begin{array}{l}\mathrm{KI}- \\
(\mathrm{n}=61)\end{array}$ & $\begin{array}{l}\mathrm{KI}+ \\
(\mathrm{n}=57)\end{array}$ & $\begin{array}{l}\mathrm{KI}- \\
(\mathrm{n}=61)\end{array}$ & $\begin{array}{l}\mathrm{Kl}+ \\
(\mathrm{n}=57)\end{array}$ & $\begin{array}{l}\mathrm{KI}- \\
(\mathrm{n}=61)\end{array}$ & $\begin{array}{l}\mathrm{KI}+ \\
(\mathrm{n}=57)\end{array}$ & $\begin{array}{l}\text { KI- } \\
(n=61)\end{array}$ \\
\hline \multirow{3}{*}{$\begin{array}{l}\text { TSH } \\
\mathrm{mU} / \mathrm{I}\end{array}$} & $\mathrm{Me}$ & 3,21 & 3,24 & 2,23 & 2,89 & 1,98 & 2,98 & 1,96 & 2,99 \\
\hline & $\begin{array}{l}25 \% \\
75 \%\end{array}$ & $\begin{array}{l}2,31 ; \\
4,19\end{array}$ & $\begin{array}{l}2,76 \\
3,98\end{array}$ & $\begin{array}{l}1,87 \\
2,54\end{array}$ & $\begin{array}{l}2,16 \\
3,78\end{array}$ & $\begin{array}{l}1,78 \\
2,16\end{array}$ & $\begin{array}{l}2,24 \\
3,78\end{array}$ & $\begin{array}{l}1,77 \\
2,01\end{array}$ & $\begin{array}{l}2,43 \\
3,98\end{array}$ \\
\hline & $\mathrm{p}$ & \multicolumn{2}{|l|}{0,328} & \multicolumn{2}{|l|}{0,021} & \multicolumn{2}{|l|}{0,001} & \multicolumn{2}{|l|}{0,001} \\
\hline \multirow{3}{*}{$\begin{array}{l}\mathrm{T} 3 \\
\mathrm{nmol} / \mathrm{I}\end{array}$} & $\mathrm{Me}$ & 3,8 & 3,9 & 3,5 & 3,7 & 3,5 & 3,6 & 3,4 & 3,6 \\
\hline & $\begin{array}{l}25 \% \\
75 \%\end{array}$ & $\begin{array}{l}3,3 ; \\
4,5\end{array}$ & $\begin{array}{l}3,3 ; \\
4,6\end{array}$ & $\begin{array}{l}3,2 ; \\
4,2\end{array}$ & $\begin{array}{l}3,2 ; \\
4,3\end{array}$ & $\begin{array}{l}2,8 ; \\
4,1\end{array}$ & $\begin{array}{l}3,2 ; \\
4,2\end{array}$ & $\begin{array}{l}2,9 ; \\
4,1\end{array}$ & $\begin{array}{l}3,2 ; \\
4,2\end{array}$ \\
\hline & $p$ & \multicolumn{2}{|l|}{0,731} & \multicolumn{2}{|l|}{0,149} & \multicolumn{2}{|l|}{0,316} & \multicolumn{2}{|l|}{0,798} \\
\hline \multirow{3}{*}{$\begin{array}{l}\text { T4 } \\
\text { nmol// }\end{array}$} & $\mathrm{Me}$ & 146,2 & 148,3 & 151,5 & 148,5 & 156,0 & 152,0 & 162,0 & 156,0 \\
\hline & $\begin{array}{l}25 \% \\
75 \%\end{array}$ & $\begin{array}{l}141,4 \\
167,2\end{array}$ & $\begin{array}{l}143,1 ; \\
168,7\end{array}$ & $\begin{array}{l}142,5 \\
165,0\end{array}$ & $\begin{array}{l}137,0 ; \\
166,5\end{array}$ & $\begin{array}{l}148,0 ; \\
167,0\end{array}$ & $\begin{array}{l}146,0 \\
168,0\end{array}$ & $\begin{array}{l}146,0 ; \\
182,0\end{array}$ & $\begin{array}{l}144,0 \\
172,0\end{array}$ \\
\hline & $p$ & \multicolumn{2}{|l|}{0,639} & \multicolumn{2}{|l|}{0,391} & \multicolumn{2}{|l|}{0,674} & \multicolumn{2}{|l|}{0,257} \\
\hline
\end{tabular}

- lodine supplementation per 6 months reduced median TSH level up to $1.96 \mathrm{mU} / \mathrm{I}$ [QR: 1,$77 ; 2,01](p<0.001)$ vs median TSH level in control group 2,99 mU/I [QR: 2,43; 3,98], $p<0.001$

- That resulted in improvement of anthropometry indicators $(92.3 \%, p<0.001)$ and psychomotor development (50\%, $p<0.001)$

- The coefficient of neuropsychological development increased (up to 92 points)

\section{Neuropsychological development (points) in children depending on iodine supplementation}

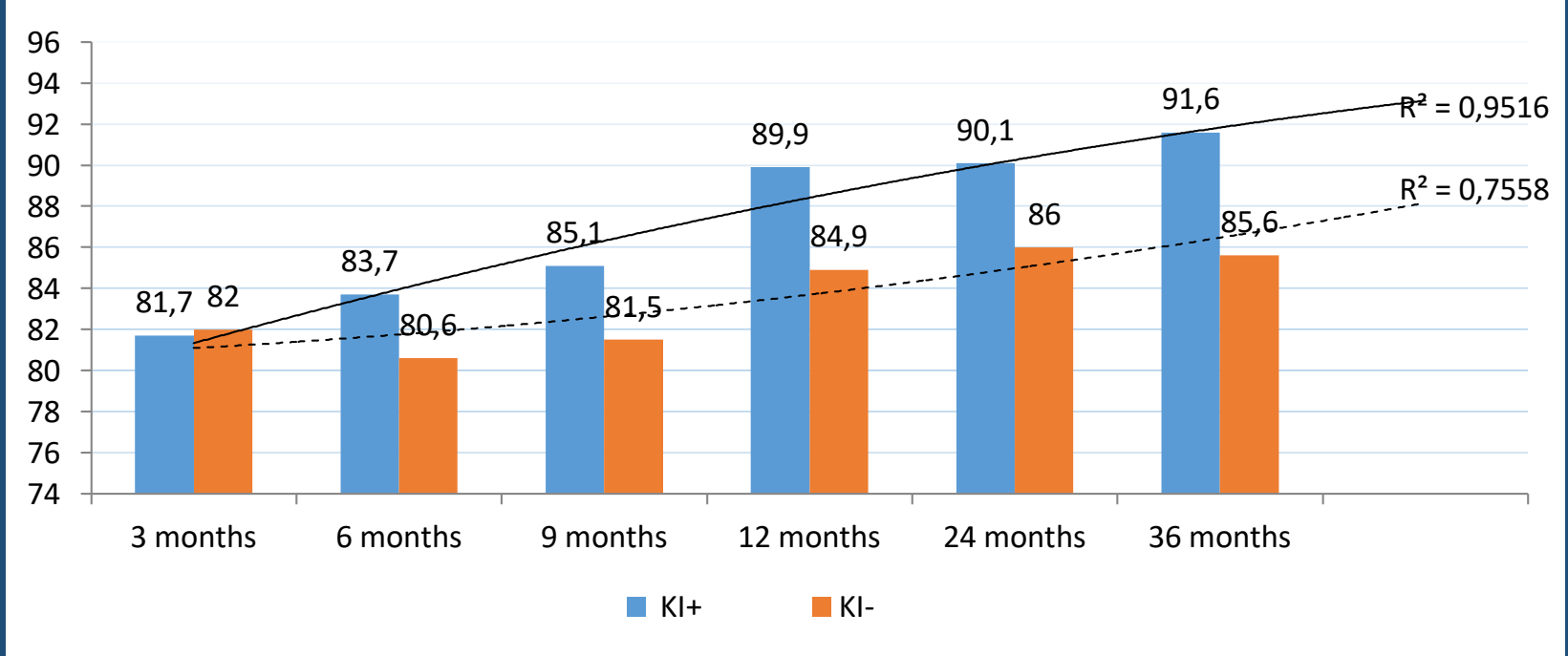

- The frequency of neurological signs reduced up to $28.9 \%$ $(p<0.001)$ 


\title{
Conclusions
}

- lodine supplementation is an effective mean of correcting physical, neuropsychological development and improvement of neurological status in children with dysfunction of the nervous system

\section{References:}

1. Costeira, M.J. Psychomotor Development of Children from an lodine-Deficient Region / M.J. Costeira, P. Oliveira, N.C. Santos [et al.] // J Pediatr. - 2011. - Vol. 159. P. 447-53.

2. Desai P. Thyroid Function in Children / M.P. Desai // Supplement To Jap. - 2011. - Vol. 59. - P. 35-59.

3. Delange F. lodine deficiency as a cause of brain damage / F. Delange // Postgrad. Med. J. 2010. - Vol. 77. - P. 217-220.

4. Desai P. Thyroid Function in Children / M.P. Desai // Supplement To Jap. - 2011. - Vol. 59. - P. 35-59.

5. Ghassabian A. Maternal Thyroid Function During Pregnancy and Behavioral Problems in the Offspring: The Generation R Study / A. Ghassabian, J.J. Bongers-Schokking, J. Henrichs [et al.] // Pediatr Res. - 2011. - Vol. 69. - P. 454-459.

6. Lazarus J.H. Antenatal Thyroid Screening and Childhood Cognitive Function / J.H. Lazarus, J.P. Bestwick, S. Channon [et al.] // N Engl J Med. - 2012. - Vol. 366 (6). P. 493-501.

7. Murcia M. Effect of lodine Supplementation During Pregnancy on Infant Neurodevelopment at 1 Year of Age / M. Murcia, M. Rebagliato, C. In iguez [et al.] // Am J Epidemiol. - 2011. - Vol. 173(7). - P. 804-812.

8. Zimmermann M.B. lodine deficiency and excess in children: worldwide status in 2013 / M.B. Zimmermann // Endocrine practice. - 2013. - Vol. 19, № 5. - P. 839-46.

\section{Presenting author:}

\author{
Hanna Shlieienkova \\ Assistant Professor, MD, PhD, \\ Department of Internal Medicine \\ V. N. Karazin Kharkiv Nacional University \\ Kharkiv, Ukraine
}

E-mail: shleen07@gmail.com 